Artículos científicos

\title{
Efecto de la adición de harina del hongo Pleurotus columbinus en la composición químico proximal y física de una pasta para la elaboración de sopas
}

Effect of the Addition of Flour of the Fungus Pleurotus Columbinus in the Proximal Chemical Composition and Physical of a Paste for the Preparation

of Soups

Efeito da adição de farinha do fungo Pleurotus columbinus na composição proximal e físico-química de uma massa para preparo de sopas

Conrado Soto Velazco Universidad de Guadalajara. Centro Universitario de Ciencias Biológicas y Agropecuarias,

Departamento de Botánica y Zoología, México conrado.soto@academicos.udg.mx https://orcid.org/0000-0002-0216-4143

Isela Alvarez Barajas Universidad de Guadalajara. Centro Universitario de Ciencias Biológicas y Agropecuarias,

Departamento de Botánica y Zoología, México leticia.alvarez@academicos.udg.mx https://orcid.org/0000-0001-5477-1546

Celia de la Mora Orozco Instituto Nacional de Investigaciones Forestales, Agrícolas y Pecuarias, México delamora.celia@inifap.gob.mx https://orcid.org/0000-0002-3675-6569 


\section{Resumen}

Las sopas son uno de los alimentos más importantes para los mexicanos. Sin embargo, por su alto contenido de carbohidratos, poca fibra y proteína, se caracterizan por estar desbalanceadas nutrimentalmente. El hongo Pleurotus incrementa la calidad nutricional de los alimentos (sopa de vegetales, snacks y pan de caja, por ejemplo). Las pastas son de bajo valor biológico por carecer de lisina; en cambio, Pleurotus columbinus posee aminoácidos esenciales, por lo que, al elaborar una pasta en combinación con este hongo, se obtendría una mayor calidad nutricional. Este trabajo muestra que la proteína de las pastas se incrementó en porcentajes de $32.15 \%$ hasta $79.27 \%$, no hubo cambio en las propiedades físicas (tiempo de cocimiento y absorción de agua), a excepción de la desintegración, que se incrementó de $6.4 \%$ hasta $8.5 \%$. La harina del hongo incrementa la cantidad proteínica de la pasta, lo cual ayudaría a mejorar la alimentación de la población.

Palabras clave: calorías, hongos comestibles, nutrientes, proteínas.

\section{Abstract}

Soups are one of the most important foods for Mexicans. However, due to their high carbohydrate content, little fiber and protein, they are characterized by being nutritionally unbalanced. The Pleurotus fungus increases the nutritional quality of food (vegetable soup, snacks, and box bread, for example). Pasta is of low biological value because it lacks lysine; on the other hand, Pleurotus columbinus has essential amino acids, which is why, when making a pasta in combination with this fungus, a higher nutritional quality would be obtained. This work shows that the protein of the pasta increased in percentages from $32.15 \%$ to $79.27 \%$, there was no change in the physical properties (cooking time and water absorption), except for disintegration, which increased from $6.4 \%$ to $8.5 \%$. The flour of the fungus increases the protein amount of the pasta, which would help improve the diet of the population.

Keywords: calories, mushrooms, nutrients, proteins. 


\section{Resumo}

As sopas são um dos alimentos mais importantes para os mexicanos. Porém, devido ao alto teor de carboidratos, pouca fibra e proteína, caracterizam-se por apresentarem desequilíbrio nutricional. O fungo Pleurotus aumenta a qualidade nutricional dos alimentos (sopa de legumes, salgadinhos e pão embalado, por exemplo). As pastas são de baixo valor biológico porque carecem de lisina; Por outro lado, Pleurotus columbinus possui aminoácidos essenciais, por isso, ao fazer uma pasta em combinação com este fungo, seria obtida uma qualidade nutricional superior. Este trabalho mostra que a proteína do macarrão aumentou em percentuais de 32,15\% para 79,27\%, não houve alteração nas propriedades físicas (tempo de cozimento e absorção de água), exceto para a desintegração, que passou de 6,4\% para 8,5\%. A farinha do fungo aumenta a quantidade de proteína da massa, o que ajudaria a melhorar a dieta alimentar da população.

Palavras-chave: calorias, cogumelos comestíveis, nutrientes, proteínas.

Fecha recepción: Diciembre 2020

Fecha aceptación: Julio 2021

\section{Introducción}

De acuerdo con el informe de la Organización de las Naciones Unidas para la Alimentación y la Agricultura [FAO, por sus siglas en inglés], las Instituciones Financieras de Desarrollo [IFD, por sus siglas en inglés], el Fondo de las Naciones Unidas para la Infancia [Unicef, por sus siglas en inglés], el Programa Mundial de Alimentos [WFP, por sus siglas en inglés] y la Organización Mundial de la Salud [WHO, por sus siglas en inglés] (2020), en los últimos cinco años se ha agudizado el hambre entre la población: decenas de millones de personas han presentado desnutrición crónica. En 2019, casi 690 millones de personas pasaron hambre, 10 millones más que en 2018. De hecho, desde 2014, 60 millones de personas se han sumado a las filas de quienes han pasado hambre. Asia tiene el mayor número

de personas desnutridas (381 millones), África ocupa el segundo lugar (250 millones), seguida de Latinoamérica y el Caribe (48 millones).

Desafortunadamente, los habitantes de países de bajos y medianos ingresos dependen en gran medida de alimentos cuya base proviene de productos vegetales y algunos derivados de los cereales. Dichos alimentos se caracterizan por poseer un bajo porcentaje de proteínas y carecer de los aminoácidos esenciales como la lisina, metionina y triptófano, los cuales son 
Revista Iberoamericana de las Ciencias Biológicas y Agropecuarias

indispensables para un buen desarrollo físico y mental adecuado en los niños y para mantener un buen rendimiento en los adultos (FAO, IFD, Unicef, WFP y WHO, 2020).

En México, un gran porcentaje de la población consume en gran medida alimentos que, si bien suelen ser económicos, o precisamente porque son baratos, son bastante calóricos, elaborados a base de cereales, raíces, tubérculos y plátanos, y en menores cantidades, alimentos costosos como la carne y lácteos (en comparación con hortalizas que son más seguras para una nutrición adecuada). En el caso particular de México, existe una disminución del consumo de frutas y lácteos a medida que aumenta la gravedad de la inseguridad alimentaria (FAO, IFD, Unicef, WFP y WHO, 2020)

Ahora bien, recientemente, por su variedad en la elaboración y su bajo costo, las pastas para sopa se han convertido en un clásico en la mayoría de los hogares mexicanos (Cardinale et al., 2005; Laboratorio Profeco Reporta, 2017). De acuerdo con la fábrica de pastas La Moderna (1 de octubre de 2019), el consumo de este alimento en México es de 3.2 kg por persona al año.

Como bien sabemos, las pastas aportan una buena cantidad de carbohidratos complejos como el almidón, que le confiere un bajo valor glucémico por su lenta absorción en el tracto digestivo. Además, se considera que su proteína es de valor biológico bajo por la deficiencia en lisina. Y por si fuera poco, tiene un escaso contenido de fibra dietética. En suma, se trata de un alimento desbalanceado (Araya, Park, Vera y Alviña, 2003). Por tal motivo, se han realizado diversos estudios encaminados a mejorar la calidad nutricional de las pastas. Uno de ellos es el de Acosta (2007), quien elaboró pastas con sémola de diferentes variedades de cebada, debido a que esta aporta un mayor contenido de lisina en comparación con la sémola de trigo. Mientras que Astaíza, Ruiz y Elizalde (2010) elaboraron pastas a partir de quinoa y zanahoria en diferentes proporciones con sémola de trigo, gracias a lo cual obtuvieron un producto de mayor calidad nutricional y de buena aceptación. Así, a partir de esta fórmula, Astaíza et al. (2010) registraron un incremento de la proteína y la fibra y una notable disminución del contenido de carbohidratos.

Otra de las alternativas que permitiría mejorar la calidad nutrimental de las pastas en particular, y en general de cualquier otro alimento, son los hongos comestibles del género Pleurotus, debido a que son una buena fuente de proteínas (11-42 g/100 g) que poseen todos los aminoácidos esenciales, entre ellos la leucina, valina, lisina, isoleucina, treonina, tirosina, metionina y fenilalanina. Son ricos en fibra dietética constituida por quitina que varía entre 11 y $31 \mathrm{~g} / 100 \mathrm{~g}$. Contienen lípidos escasos, poseen ácidos grasos esenciales, tal como el ácido 
oleico y linoleico; vitaminas del complejo B, y en lo que respecta a los minerales, se ha encontrado fósforo, potasio, hierro, calcio, magnesio, manganeso y cobre, así como un bajo valor energético. Además, poseen polisacáridos de 36 a 60 g/100 g, entre ellos alfa y betaglucanos, que han demostrado tener efectos inmunomodulantes (Asaduzzaman y Mousumi, 2012; Rampinelli et al., 2010; Vega y Franco, 2012).

Pleurotus columbinus se cultiva en diversas regiones de México (Guzmán et al., 2008; Soto y Arias 2004) y se comercializa en supermercados, mercados y tianguis, por lo que tiene un potencial muy alto para ser aprovechado como un fortificador de alimentos, tal y como se ha demostrado con sopa de vegetales, snacks, galletas y pan de caja (Ghorai et al., 2009; Lavelli, Proserpio, Gallotti, Laureati y Pagliarini, 2018; Parab, Dhalagade, Sahoo y Ranveer, 2012; Proserpio, Lavelli, Laureati y Pagliarini, 2019; Ng, Wan Amir Nizam y Wan Rosli, 2017). Localmente, Soto et al., (2005) realizaron un estudio respecto a su calidad nutrimental y encontraron valores de proteína de 29.93 g/100 g., 15 aminoácidos esenciales, fibra $11.7 \mathrm{~g} / 100 \mathrm{~g}$, lípidos $1.43 \mathrm{~g} / 100 \mathrm{~g}$ y cenizas de $8.94 \mathrm{~g} / 100 \mathrm{~g}$. De aquí que en este trabajo se plantea la incorporación de harina de las fructificaciones de este hongo a una pasta para sopa tipo tallarín, por ser un alimento consumido popularmente por la mayoría de la población. Con este trabajo se pretende obtener un alimento de mejor calidad nutrimental y más que nada proteínica que a la larga prevenga enfermedades, tales como las cardiovasculares, obesidad, diabetes tipo dos y cáncer.

\section{Materiales y método}

El presente estudio se desarrolló en el Laboratorio de Cultivo de Hongos Comestibles del Departamento de Botánica y Zoología de la Universidad de Guadalajara. Las pastas se elaboraron de acuerdo con la norma oficial mexicana NMX-F-023-NORMEX-2002 (Secretaría de Economía, 23 de septiembre de 2002), clasificación tipo III: pasta de harina de trigo o semolina con vegetales para sopa (tallarines). Y en este caso, se utilizó harina de Pleurotus columbinus (HPc).

La semolina (S) fue brindada por la empresa La Moderna, S. A. de C. V., de Zapopan, Jalisco. Las fructificaciones frescas de P. columbinus se cosecharon de un substrato de rastrojo de maíz, tal como lo describen Soto y Arias (2004). Estas se deshidrataron en una secadora de madera diseñada para tal fin, a una temperatura de $40^{\circ} \mathrm{C}$. Una vez secas se 
Revista Iberoamericana de las Ciencias Biológicas y Agropecuarias

pulverizaron en un molino de cuchillas Cyclone Mill (Retsch Twister X 100) con una criba de $0.3 \mathrm{~mm}$.

A ambos tipos de harinas, $\mathrm{S}$ y $\mathrm{H} P c$, se les hicieron análisis químico-proximales de acuerdo con lo establecido por la Asociación Oficial de Química Analítica [AOAC, por sus siglas en inglés] (1990). Dichos análisis se realizaron en el Laboratorio de Bioingeniería del Departamento de Madera Celulosa y Papel de la Universidad de Guadalajara. Se hicieron las determinaciones de ceniza (AOAC 7-010/70), fibra cruda (FC) y extracto etéreo (EE) (AOAC 7-048/70). En cuanto a la proteína cruda (PC), se utilizó el método Microkjeldahl (AOAC 42-014/70). El valor del nitrógeno total obtenido se multiplicó por el factor de corrección de 5.75, en el caso de la S, y para la HPc se utilizó el factor de corrección de $\mathrm{N} \times$ 4.38 (Lau, 1983; Soto, Serratos, Ruiz y García, 2005). El extracto libre de nitrógeno (ELN) se determinó por diferencia entre el total de sólidos y la suma de las determinaciones anteriores. Por último, se calcularon las kcal/100 de cada ingrediente de $\mathrm{S}: \mathrm{PC} \times 4, \mathrm{ELN} \times 4$ y EE $\times 9$. En el caso de la $\mathrm{HPc}$, se utilizaron los factores propuestos por Mattila, Salo, Konko, Aro y Jalava (2002): PC × 2.62, $\mathrm{EE} \times 8.37$ y $\mathrm{ELN} \times 3.48$.

Las pastas se elaboraron en las siguientes proporciones (S:Pc): 100:00, 90:10, 85:15, 80:20 y 70:30. Las harinas se revolvieron manualmente hasta obtener una mezcla homogénea. Posteriormente, a cada muestra se le adicionó agua potable hasta obtener una masa con la consistencia adecuada de amasado. El amasado se efectuó manualmente y la masa obtenida se dejó reposar a temperatura ambiente durante unos 50 minutos. Con una máquina Olimpia tipo Luso modelo 150, a la masa se le dio forma laminar hasta obtener un grosor de lámina de $\pm 1 \mathrm{~mm}$ característico de un tallarín. Dicha lámina se cortó con ayuda de un rodillo con cuchillas propio de la máquina para la obtención final de los tallarines.

El secado de los tallarines se realizó en tres etapas con el objetivo de reducir el contenido de humedad a menos de $12 \%$. El primer paso consistió en un presecado durante el cual los tallarines se mantuvieron durante dos horas a una temperatura entre 30 y $40{ }^{\circ} \mathrm{C}$, con el fin de disminuir la actividad microbiológica o enzimática, endurecer el gluten periférico, fortificar la estructura de la pasta y reducir el tiempo del periodo final del secado.

El siguiente paso fue una pasteurización: los tallarines se mantuvieron a $60{ }^{\circ} \mathrm{C}$ durante 30 minutos. El objetivo de esta etapa fue conseguir el equilibrio de humedad entre el interior de la pasta y su superficie, ya que en el presecado la humedad se aleja del interior de la pasta a través de los capilares en la misma proporción en que la humedad se evapora de la superficie, pero luego esta proporción decrece y la humedad comienza a concentrarse 
Revista Iberoamericana de las Ciencias Biológicas y Agropecuarias

alrededor del centro de la pasta, lo que hace necesario equilibrar la humedad interior y exterior.

La etapa final de secado fue a una temperatura entre 45 y $55^{\circ} \mathrm{C}$ durante cuatro horas. En este periodo se trató de eliminar la mayor cantidad de agua posible para obtener un producto con un máximo de $12 \%$ de humedad. Finalmente, la pasta se dejó a temperatura ambiente durante $12 \mathrm{~h}$, para posteriormente empacarla en bolsas de polipropileno para su uso en los análisis siguientes. Se considera que, aproximadamente, la mitad del agua es absorbida por la proteína. Esta última es más difícil de remover, de ahí los largos períodos de secado.

Se tomaron muestras representativas de las pastas, las cuales se pulverizaron con ayuda de un molino eléctrico hasta permitir el paso del pulverizado a través de una criba de $1 \mathrm{~mm}$. Posteriormente, se realizaron las siguientes determinaciones: ceniza, FC, EE, PC y ELN, de acuerdo con los métodos descritos anteriormente. Asimismo, se realizó el análisis a una pasta comercial (Rex) tipo tallarín, sin huevo, como testigo. Una vez obtenidas las determinaciones, se calculó el contenido energético como fue descrito anteriormente.

Las propiedades físicas de una pasta permiten delimitar sus características para establecer un juicio de calidad, por lo que a los tallarines se les realizaron los siguientes análisis físicos, siguiendo aquí a Acosta (2007):

- Volumen en pasta cruda: se pesó $100 \mathrm{~g}$ de muestra y se colocó en un cilindro de malla fina, el cual se introdujo en una probeta de $1000 \mathrm{ml}$ con aproximadamente $800 \mathrm{ml}$ de agua y se midió el líquido desalojado.

- $\quad$ Tiempo de cocimiento: a un litro de agua en ebullición, se adicionaron $100 \mathrm{~g}$ de muestra y $10 \mathrm{~g}$ de cloruro de sodio y se midió el tiempo requerido para el cocimiento de la pasta.

- Peso de la pasta cocida: una vez obtenido el tiempo de cocción, la pasta se drenó durante 10 minutos con ayuda de un embudo y en seguida se pesó en húmedo.

- Grado de absorción de agua: es la diferencia obtenida entre el peso de pasta cocida y el peso de pasta cruda, se estableció como mínimo 150 g de aumento.

- Volumen en pasta cocida: al igual que para el volumen en pasta cruda, la pasta cocida se colocó en un cilindro de malla fina, se introdujo en una probeta de $1000 \mathrm{ml}$ con $800 \mathrm{ml}$ de agua y se midió el aumento de volumen de agua. 
- $\quad$ Porcentaje de aumento de pasta cocida con respecto a pasta cruda: para esta determinación se realizó el siguiente cálculo: V1 - V2/V2 × 100, donde: $V 1=$ volumen de pasta cocida y $V 2=$ volumen de pasta cruda

El porcentaje de sedimentación o desintegración se realizó con 100 g de pasta, la cual se coció en $1000 \mathrm{ml}$ de agua durante 10 minutos, el líquido se drenó y la pasta se colocó en una probeta de $1000 \mathrm{ml}$. Se midió el sedimento formado en mililitros y se calculó como porcentaje. Se estableció $20 \%$ de sedimentación como máximo.

\section{Diseño experimental}

En todos los casos, se siguió un diseño completamente al azar con un arreglo de $6 \times 6$ con tres repeticiones para los tratamientos. Así, se contemplaron seis tipos de pasta y seis determinaciones nutrimentales. A los resultados se les aplicó un análisis de varianza (Anova) de un solo factor para determinar si existían diferencias estadísticas significativas. Para la separación de grupos, se utilizó la prueba de Tukey con una $p<0.05$ y $95 \%$ de nivel de confianza.

\section{Resultados y discusión}

\section{Análisis químicos proximales}

Los análisis químicos proximales realizados a las materias primas para la elaboración de las pastas en este estudio, $\mathrm{S}$ y $\mathrm{HPc}$, se presentan en la tabla 1. La PC que se determinó para la S fue de $11.34 \%$ y para la harina del hongo de $30.1 \%$. El EE con 0.93 y $1.8 \%$ para la $\mathrm{S}$ y la $\mathrm{HPc}$, respectivamente. La FC que se encontró en la $\mathrm{S}$ fue de $0.26 \%$ y en la harina del hongo de $10.4 \%$. El porcentaje de cenizas en la S correspondió a $0.6 \%$ y para la HPC $10 \%$. Por último, el extracto libre de nitrógeno encontrado en la S es de $86.87 \%$ y de $47.7 \%$ en la HPc. Cabe indicar que tabla 1 también se presentan los datos obtenidos en la pasta comercial Rex empleada como testigo. 
Tabla 1. Análisis químico proximal de los ingredientes utilizados para hacer los tallarines y comparados con una pasta comercial. Valores promedio $(N=9)$

\begin{tabular}{|c|c|c|c|}
\hline Nutrimento (\%) & Semolina & $\begin{array}{c}\text { Harina de } \\
\text { P. columbinus }\end{array}$ & $\begin{array}{c}\text { Pasta comercial } \\
\text { (Rex) }\end{array}$ \\
\hline Proteína cruda & 11.34 & 30.1 & 9.61 \\
\hline Extracto etéreo & 0.93 & 1.8 & 1.66 \\
\hline Fibra cruda & 0.26 & 10.4 & 0.09 \\
\hline Cenizas & 0.6 & 10 & 0.8 \\
\hline $\begin{array}{c}\text { Extracto libre de } \\
\text { nitrógeno }\end{array}$ & 86.87 & 47.7 & 87.84 \\
\hline Kcal/100 & 401.21 & 260 & 404.74 \\
\hline
\end{tabular}

Fuente: Elaboración propia

En tabla 2, por su parte, se muestran los resultados obtenidos de los análisis químicos proximales de los tallarines elaborados con base en las mezclas de $\mathrm{S}$ y la $\mathrm{H} P c$, así como los determinados en una pasta comercial (Rex) tipo tallarín, sin huevo. Allí se observa que, conforme se incrementa la cantidad de HPc, se incrementa la cantidad de PC: de $10.95 \%$ (S) a $19.63 \%$ con la mezcla 70:30 (+79.27\%) ( $p>0.05)$. Asimismo, es de hacer notar que el contenido energético fue muy similar en las mezclas con la muestra control. 
Revista Iberoamericana de las Ciencias Biológicas

y Agropecuarias

Tabla 2. Se muestran los análisis de las diferentes proporciones de las mezclas de S y HPc, así como la pasta control y el testigo comercial. Valores promedio $(N=18)$

\begin{tabular}{|c|c|c|c|c|c|c|}
\hline & & \multicolumn{5}{|c|}{ Proporciones: $\mathrm{S}: \mathrm{H} P c$} \\
\hline $\begin{array}{c}\text { Nutrimento } \\
\%\end{array}$ & $\begin{array}{l}\text { Pasta } \\
\text { Rex }\end{array}$ & $S$ & $90: 10$ & $85: 15$ & $80: 20$ & $70: 30$ \\
\hline $\begin{array}{l}\text { Proteína } \\
\text { cruda } \\
(\mathrm{N} \times 5.76)\end{array}$ & $9.61^{\mathrm{e}}$ & $10.95^{\mathrm{d}}$ & $14.47^{\mathrm{c}}$ & $16.70^{\mathrm{b}}$ & $17.50^{\mathrm{b}}$ & $19.63^{\mathrm{a}}$ \\
\hline $\begin{array}{l}\text { Extracto } \\
\text { etéreo }\end{array}$ & $1.66^{\mathrm{ns}}$ & $0.90^{\mathrm{ns}}$ & $1.06^{\mathrm{ns}}$ & $1.05^{\mathrm{ns}}$ & $1.16^{\mathrm{ns}}$ & $1.26^{\mathrm{ns}}$ \\
\hline Fibra cruda & $0.09^{\mathrm{b}}$ & $0.20^{\mathrm{a}}$ & $0.093^{b}$ & $0.21^{\mathrm{a}}$ & $0.21^{\mathrm{a}}$ & $0.21^{\mathrm{a}}$ \\
\hline Cenizas & $0.80^{\mathrm{d}}$ & $0.65^{d}$ & $2.33^{c}$ & $2.75^{b}$ & $2.86^{\mathrm{b}}$ & $3.06^{\mathrm{a}}$ \\
\hline $\begin{array}{l}\text { Extracto } \\
\text { libre de } \\
\text { nitrógeno }\end{array}$ & $87.84^{d}$ & $87.3^{d}$ & $82.04^{c}$ & $79.29^{b}$ & $78.30^{b}$ & $75.84^{a}$ \\
\hline Kcal/100 & $404.74^{\mathrm{ns}}$ & $401.1^{\mathrm{ns}}$ & $395.58^{\mathrm{ns}}$ & $389.33^{\mathrm{ns}}$ & $383.8^{\mathrm{ns}}$ & $393.22^{\mathrm{ns}}$ \\
\hline
\end{tabular}

Fuente: Elaboración propia

En la figura 1 se indican los porcentajes de PC encontrados en cada una de las mezclas. En la mezcla 90:10, fue de $14.47 \%$; en la de 85:15, de $16.70 \%$; en la de 80:20, de $17.50 \%$, y en la de 70:30, de $19.63 \%$. La pasta elaborada únicamente con $\mathrm{S}$ tuvo un porcentaje de PC de $10.95 \%$ y la pasta comercial presentó un valor de $9.61 \%$. Las pastas para sopa, por lo general, poseen alrededor del 10-15 \% de proteína cruda. Con la adición de la harina del hongo, se observó un incremento considerable en el contenido proteínico de las pastas: desde un $32.15 \%$ hasta $79.27 \%$ en la proporción 90:10 y 70:30, respectivamente. Si se toma en cuenta que una persona adulta requiere un consumo diario de $75 \mathrm{~g}$ de proteína (Serralda, Meléndez y Pasquetti, 2003), una dieta con pastas fortificadas con HPc podría cubrir de $50 \%$ a $100 \%$ de los requerimientos diarios. Por otro lado, está comprobado que la mayoría de los cereales, y particularmente el trigo, son deficientes en el aminoácido lisina, por lo que la adición de la HPc a las pastas para sopa incrementaría sustancialmente el contenido de este y otros aminoácidos (Soto et al., 2005) 
Figura 1. Se esquematizan los resultados de PC encontrados en las mezclas de S y HPc. Se observa un incremento marcado cuando se añade más harina de hongos

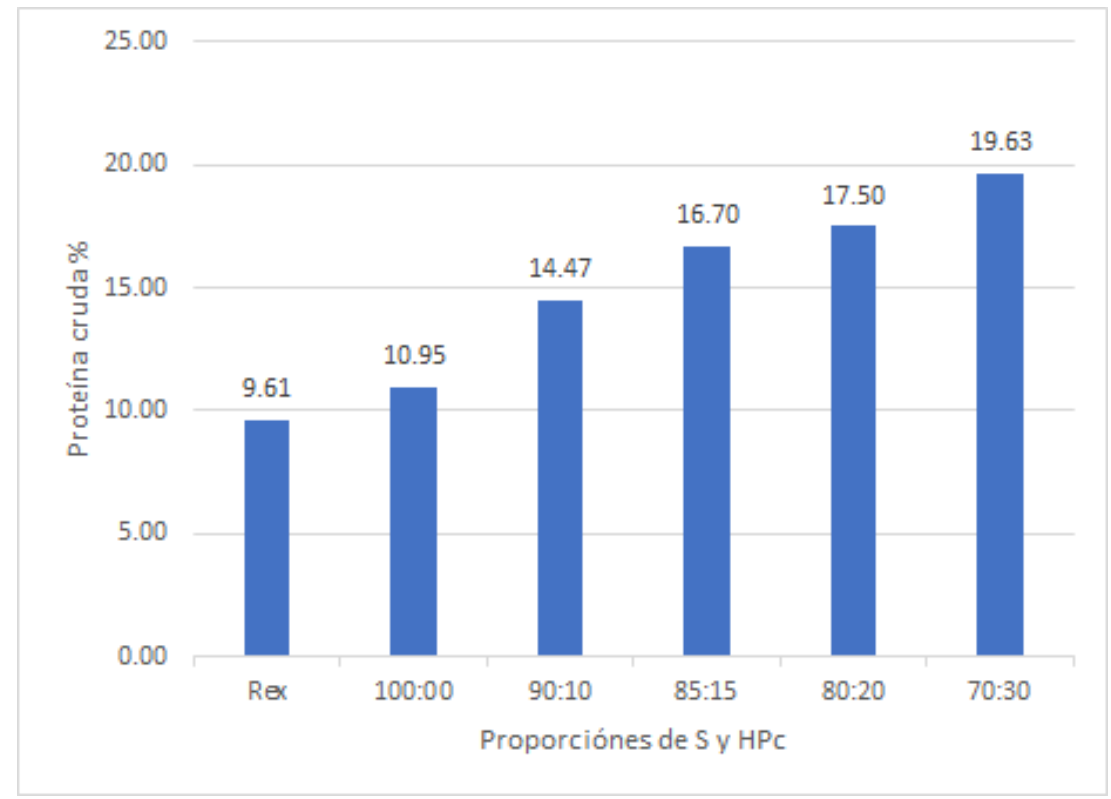

Fuente: Elaboración propia

Con los datos de proteína que se determinaron en todas las muestras, se encontró una correlación positiva entre la adición de la HPc y la cantidad de proteína obtenida, como se muestra en la figura 2.

Figura 2. Indica la relación positiva que tiene la adición de $\mathrm{H} P c$ a la S para el incremento de la proteína de las pastas elaboradas con estos ingredientes

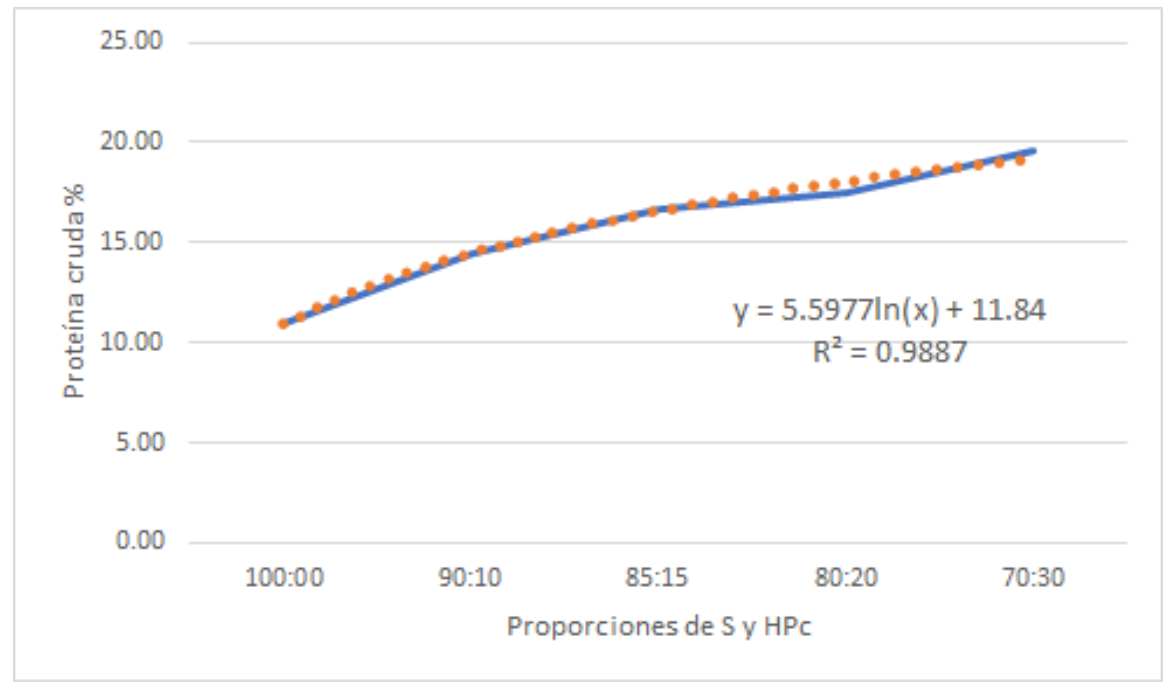

Fuente: Elaboración propia 
La determinación de EE de las pastas se observa en la figura 3. En general, los porcentajes que se obtuvieron oscilaron entre $1.26 \%$ y $1.05 \%$ para todas las mezclas de S y $\mathrm{H} P$ c. La pasta comercial y la pasta de S tuvieron $1.66 \%$ y $0.9 \%$, respectivamente. El EE en las mezclas se determinó en un intervalo de $1.06 \%$ (90:10) y $1.26 \%$ (70:30).

Figura 3. Los valores de EE permanecieron sin mucha alteración comparados con la pasta Rex y la $S$

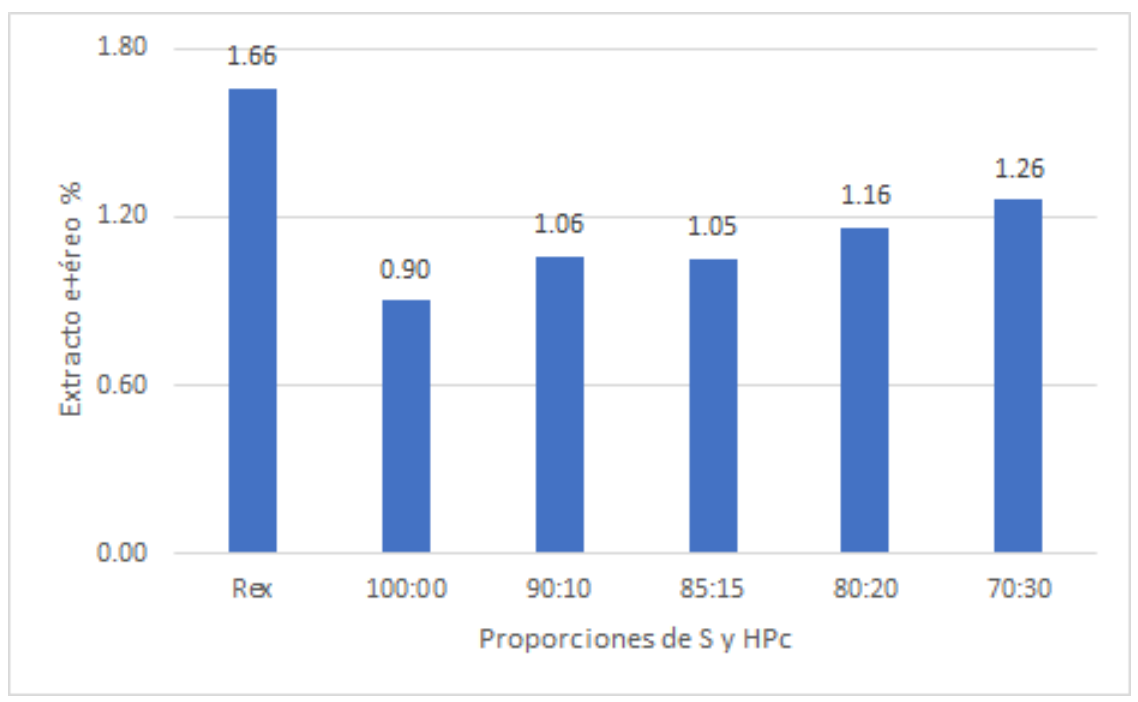

Fuente: Elaboración propia

En cuanto al contenido de FC en la mezcla 90:10, fue de 0.10\%; en las muestras restantes $(85: 15,80: 20$ y 70:30) de $0.21 \%$. En la pasta elaborada con $\mathrm{S}$ fue de $0.2 \%$ y la pasta comercial de $0.09 \%$ (figura 4). En cuanto al contenido de fibra cruda, la pasta elaborada a base de S a $100 \%$ y la pasta comercial presentaron valores muy bajos, de $0.09 \%$, no así las pastas elaboradas con mezclas de las harinas, cuyo valor fue estadísticamente diferente con $0.21 \%$. 
Figura 4. Se indican las determinaciones para el contenido de FC. Los valores fueron muy semejantes entre la muestra testigo y la $\mathrm{S}$, pero diferentes en relación con las pastas adicionadas con harina del hongo

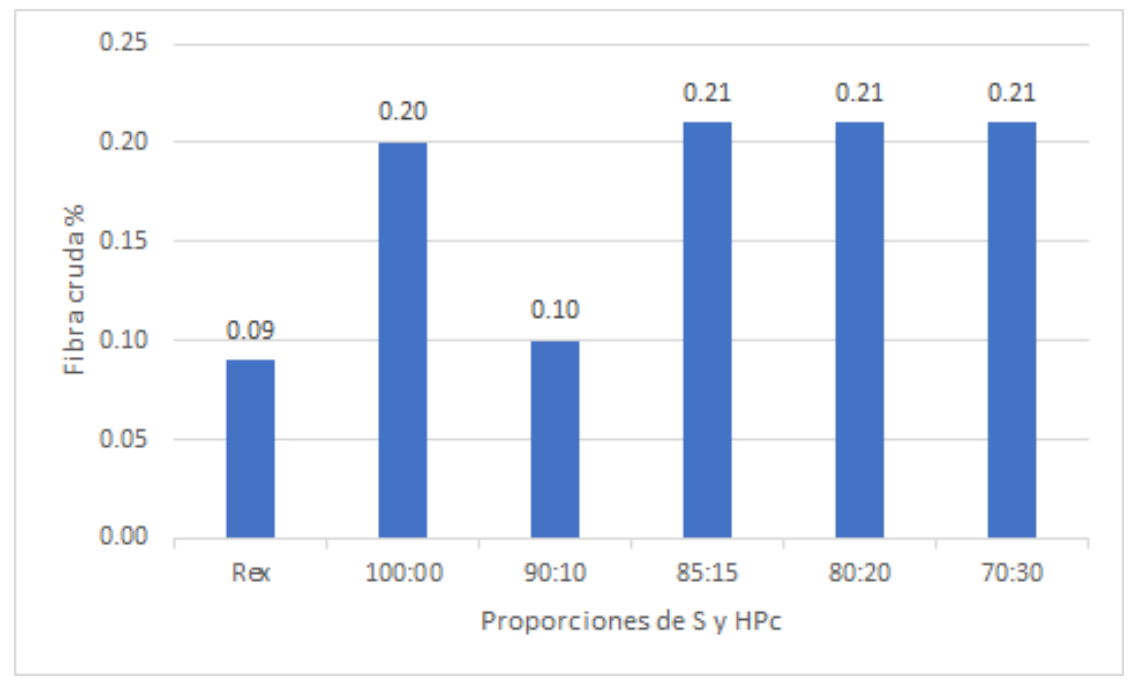

Fuente; Elaboración propia

Los porcentajes de cenizas determinados en las muestras se presentan en la figura 5. En general, en las mezclas se encontraron porcentajes alrededor del $2.33 \%$ y $3.06 \%$. En la pasta 90:10, el porcentaje fue de $2.33 \%$; en $85: 15$, de $2.75 \%$; en $80: 20$, de $2.86 \%$, y de $3.06 \%$ en la proporción 70:30. En la pasta elaborada con S se obtuvo un valor de $0.65 \%$ y en la pasta comercial de $0.8 \%$. Se encontró que la $\mathrm{H} P c$ incrementa la cantidad de cenizas $(258.2 \%$ a $371 \%)$ conforme se mezcla con la S. 
Figura 5. Las cenizas tuvieron un aumento en las pastas elaboradas con $\mathrm{H} P c$ en relación con la pasta Rex y de $\mathrm{S}$

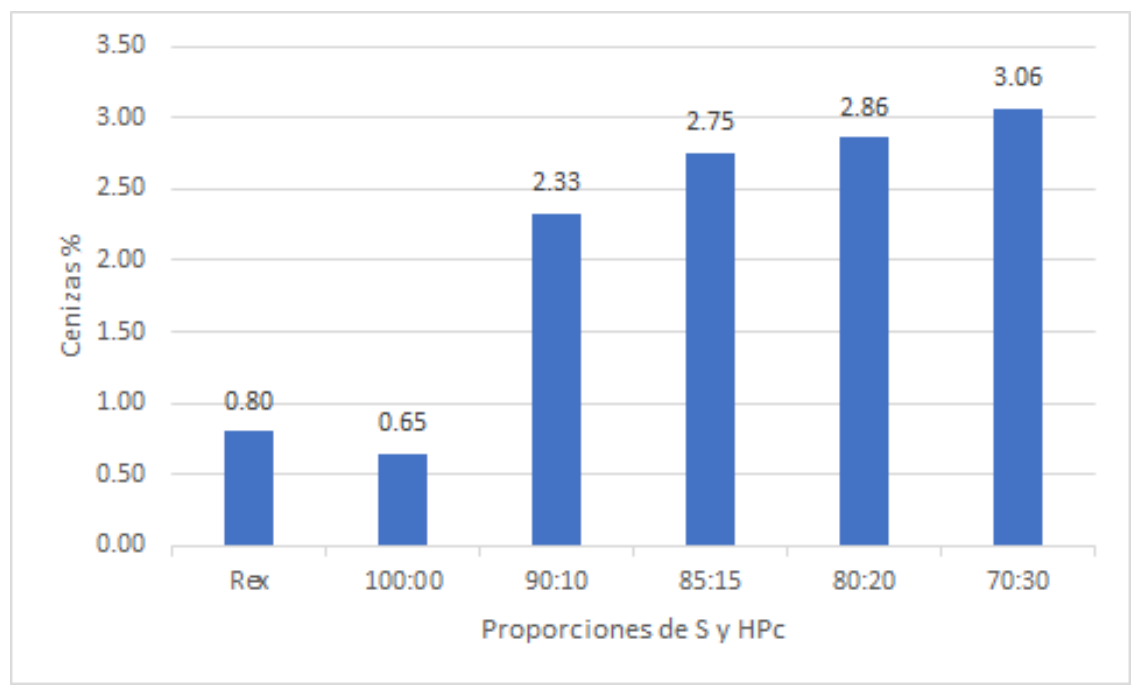

Fuente: Elaboración propia

El ELN tuvo valores altos en todas las muestras estudiadas. La figura 6 muestra estos resultados. En la mezcla 90:10, se obtuvo un porcentaje de 82.04 \%. Con la mezcla de 85:15, se encontró $79.29 \%$. En la de 80:20, de $78.3 \%$ y de $75.84 \%$ en la de 70:30. En la pasta de S fue un porcentaje de $87.3 \%$ y con la pasta comercial se determinó $87.84 \%$. Sin embargo, el contenido de ELN disminuyó (-14.10 \%) conforme se aumentó la inclusión de la harina del hongo. Esto puede ser importante desde el punto de vista nutrimental, ya que esta disminución de carbohidratos representa una disminución de calorías, lo cual es benéfico para un mejor aprovechamiento de las proteínas. 
Revista Iberoamericana de las Ciencias Biológicas y Agropecuarias

Figura 6. El ELN en las pastas Rex y semolina poseen un mayor porcentaje de solubles que las elaboradas con $\mathrm{HPC}$

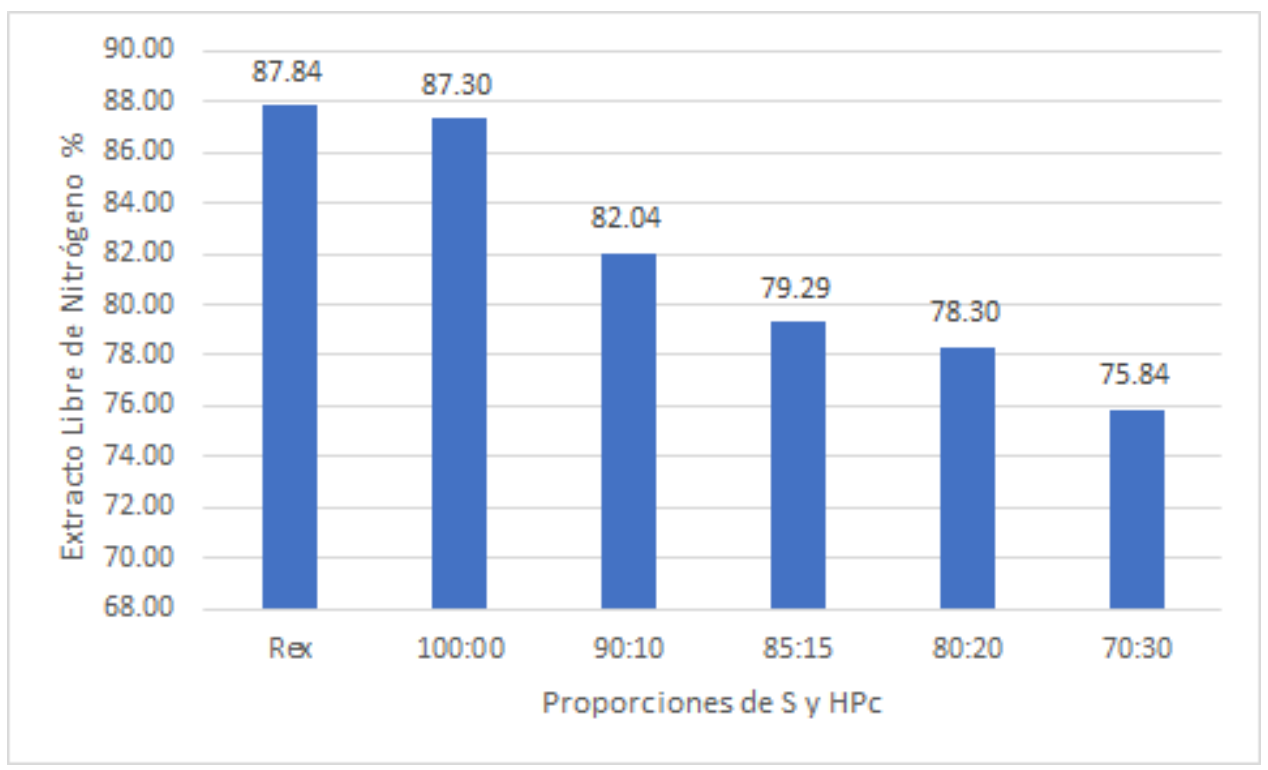

Fuente: Elaboración propia

\section{Pruebas físicas}

En la tabla 3 se observa el peso de la pasta una vez que fue cocida durante 10 minutos en agua hirviendo. La pasta elaborada con $100 \%$ de S tuvo un peso final de $230 \mathrm{~g}$, lo cual indica que absorbió $130 \mathrm{~g}$ de agua y un aumento de peso de 2.3 veces. La pasta comercial Rex pesó 249 g, de los cuales 149 g correspondieron al agua absorbida, esto es, un aumento de 2.49 veces. Las pastas elaboradas a base de S y HPc tuvieron una menor absorción de agua, por ejemplo, la mezcla 90:10 y 85:15 tuvieron un peso de 180 g, que indica una retención de agua en la pasta cocida de $80 \mathrm{~g}$ (1.8 veces). La mezcla de 80:20 pesó en pasta cocida $187 \mathrm{~g}$, de los cuales $87 \mathrm{~g}$ correspondieron al agua absorbida (1.87 veces). La proporción 70:30 en pasta cocida pesó 193 g, en donde 93 g fueron de agua absorbida, con un aumento de peso de 1.93 veces. De acuerdo con las normas de calidad de las pastas para sopas, la harina del hongo no afectó el tiempo de cocimiento, ya que, en general, solo bastaron 10 minutos para el ablandamiento de las pastas. El mismo tiempo de cocimiento se observó con la pasta elaborada con pura S y la pasta comercial.

Para que una pasta se considere de buena calidad, es necesario que aumente dos veces su peso una vez cocida. En la tabla 3 se observa que solo la pasta de S y la comercial aumentaron el doble de peso; ninguna de las pastas elaboradas con las mezclas de harina 
Revista Iberoamericana de las Ciencias Biológicas y Agropecuarias

dobló su peso, aunque su diferencia es solo de 0.20 y 0.07 unidades, por lo que esa falta se consideró insignificante.

Respecto al grado de absorción de agua (tabla 3), las pastas elaboradas con las mezclas de harinas tuvieron una menor absorción en relación con las de $\mathrm{S}$ y la pasta comercial. Sin embargo, la pasta de S ni la comercial alcanzaron la cantidad mínima de 150 g, como lo establecen las normas oficiales. En este aspecto, la harina del hongo sí afecta negativamente la absorción de agua.

Tabla 3. Se indica el aumento de peso de la pasta cocida en relación con la pasta cruda, así como el agua absorbida. Valores promedio $(N=15)$

\begin{tabular}{|c|c|c|c|c|c|}
\hline $\begin{array}{c}\text { Pasta } \\
\text { S:HPc }\end{array}$ & $\begin{array}{c}\text { Peso en } \\
\text { pasta cruda } \\
(\mathrm{g})\end{array}$ & $\begin{array}{c}\text { Peso en } \\
\text { pasta cocida } \\
(\mathrm{g})\end{array}$ & $\begin{array}{c}\text { Aumento de } \\
\text { peso }(\mathrm{g})\end{array}$ & $\begin{array}{c}\text { Tiempo de } \\
\text { cocción } \\
(\mathrm{min})\end{array}$ & $\begin{array}{c}\text { Agua } \\
\text { absorbida } \\
(\mathrm{g})\end{array}$ \\
\hline $100: 00$ & 100 & $230^{\mathrm{b}}$ & 2.30 & 10 & $130^{\mathrm{a}}$ \\
\hline $90: 10$ & 100 & $180^{\mathrm{d}}$ & 1.80 & 10 & $80^{\mathrm{d}}$ \\
\hline $85: 15$ & 100 & $180^{\mathrm{d}}$ & 1.80 & 10 & $80^{\mathrm{d}}$ \\
\hline $80: 20$ & 100 & $187^{\mathrm{cd}}$ & 1.87 & 10 & $87^{\mathrm{cd}}$ \\
\hline $70: 30$ & 100 & $193^{\mathrm{c}}$ & 1.93 & 10 & $93^{\mathrm{c}}$ \\
\hline Rex & 100 & $249^{\mathrm{a}}$ & 2.49 & 10 & $149^{\mathrm{b}}$ \\
\hline Nota: letras distintas en la misma columna muestran diferencias estadísticamente \\
\multicolumn{7}{|r|}{ significativas. } \\
\hline
\end{tabular}

Fuente: Elaboración propia

En cuanto al aumento de volumen que se considera como una norma, debe ser de tres a cuatro veces el original. La tabla 4 muestra el aumento del volumen de la pasta cruda con respecto a la pasta cocida. Ninguna de las pastas elaboradas con las mezclas de harinas alcanzó ese aumento. Del mismo modo, la pasta de S y la pasta comercial no obtuvieron ese volumen. A esta última, solo le faltaron 0.20 unidades.

Otro de los factores que se consideran importantes en la calidad de una pasta es la cantidad de sedimentación o desintegración que, de acuerdo con las normas, se establece en $20 \%$ como máximo. En la tabla 4 se puede apreciar que en ninguno de los casos se rebasa el límite permitido, aunque, conforme se aumentó la cantidad de HPc a la mezcla, se incrementó el porcentaje de sedimento. 
Es conveniente señalar que existen pastas para sopa comerciales a las que se les adiciona algún tipo de adhesivo para mantener bajo el nivel de desintegración, como, por ejemplo, la clara de huevo. En el caso de las pastas de este estudio, no se agregó ningún aditivo, por lo que sería conveniente utilizar alguno de ellos para eliminar este problema.

En la tabla 4 se indican los resultados en relación con el volumen en pasta cruda, pasta cocida y el porcentaje de aumento, así como el porcentaje de desintegración de las pastas en estudio. La pasta elaborada con $100 \%$ de S tuvo un porcentaje de aumento de $137.5 \%$; sin embargo, la pasta comercial lo tuvo de $182 \%$. En las mezclas de S y HPc, el porcentaje de aumento en la mezcla de 90:10 fue de $114.2 \%$ y en la mezcla de 85:15 fue de 106.2 \%. Las mezclas de 80:20 y 70:30 tuvieron el mismo porcentaje de aumento de 112.5.

Respecto al porcentaje de sedimentación o desintegración de las pastas, se encontró que la pasta de S y la comercial presenta alrededor de $5 \%$ de desintegración. Las pastas con mezclas de harinas presentaron un porcentaje de desintegración más alto, de $6.4 \%$ hasta $8.5 \%$, conforme se aumentó la cantidad de HPc.

Tabla 4. Se muestran los resultados del aumento de volumen de la pasta cruda y la cocida, así como el porcentaje de sedimentación. Valores promedio $(N=15)$

\begin{tabular}{|c|c|c|c|c|c|}
\hline $\begin{array}{l}\text { Pasta } \\
\text { S:HPc }\end{array}$ & $\begin{array}{c}\text { Volumen } \\
\text { (100 g) } \\
\text { pasta } \\
\text { cruda } \\
(\mathrm{mL})\end{array}$ & $\begin{array}{c}\text { Volumen } \\
\text { pasta } \\
\text { cocida } \\
(\mathrm{mL})\end{array}$ & $\begin{array}{l}\text { Aumento } \\
\text { de } \\
\text { volumen } \\
\text { (veces) }\end{array}$ & $\begin{array}{c}\text { Aumento } \\
(\%)\end{array}$ & $\begin{array}{c}\text { Sedimentos } \\
(\%)\end{array}$ \\
\hline 100:00 & 80 & $190^{b}$ & 2.4 & 137.5 & $5^{\mathrm{e}}$ \\
\hline $90: 10$ & 70 & $150^{d}$ & 2.1 & 114.2 & $6.4^{\mathrm{cd}}$ \\
\hline $85: 15$ & 80 & $165^{c}$ & 2.0 & 106.2 & $6.8^{b c}$ \\
\hline $80: 20$ & 80 & $170^{\mathrm{c}}$ & 2.1 & 112.5 & $7.5^{\mathrm{ab}}$ \\
\hline $70: 30$ & 80 & $170^{\mathrm{c}}$ & 2.1 & 112.5 & $8.5^{\mathrm{a}}$ \\
\hline $\operatorname{Rex}$ & 78 & $220^{\mathrm{a}}$ & 2.8 & 182 & $5.5^{\mathrm{de}}$ \\
\hline \multicolumn{6}{|c|}{ Nota: letras distintas en la misma columna muestran diferencias } \\
\hline
\end{tabular}

Fuente: Elaboración propia 
Un aspecto que no se mencionó en los resultados, pero que debe de tomarse en cuenta, es el cambio de color de las pastas. Las pastas de S y la comercial son de color blanco amarillento; en cambio, las pastas elaboradas con S y HPc gradualmente, conforme aumenta el porcentaje de inclusión, cambian de blanco amarillento a color café claro. Dicho cambio de color podría afectar la aceptabilidad y preferencia de estas pastas en el gusto de la gente, por lo que, como un seguimiento a este estudio, sería conveniente realizar análisis sensoriales y estudios de aceptabilidad de estos tallarines. Dichos estudios conllevarían realizar aislados proteínicos de los hongos, para incluirlos en las pastas, así como el decolorar y desodorizar previamente la $\mathrm{HPc}$.

\section{Conclusión}

Se puede decir que la $\mathrm{H} P c$ incrementa el porcentaje de proteína de las pastas en relación con las elaboradas con semolina. Asimismo, la FC, ELN y las cenizas tienen un aumento significativo. Los lípidos y las kilocalorías no mostraron cambios en relación con el control y el testigo. Por otro lado, las propiedades físicas se alteran poco en relación con las pastas comerciales, a excepción del cambio de color conforme se incrementa el porcentaje de inclusión de $\mathrm{HPc}$. Es necesario realizar estudios de aceptabilidad.

\section{Futuras líneas de investigación}

Los hongos comestibles Pleurotus tienen un potencial como fortificadores de alimentos, por lo que es indispensable continuar con este estudio respecto a la calidad nutrimental que tienen las sopas adicionadas con la harina de este hongo. El paso siguiente y a corto plazo es determinar los aminoácidos esenciales que se integraron a esta pasta. Además, es necesario el estudio sensorial, para determinar el grado de aceptabilidad de este

alimento tomando como punto de partida el cambio de color de las pastas. Por otra parte, buscar la vinculación de este producto a la industria de producción de pastas alimenticias con la finalidad de poder ofertar esta idea. 


\section{Referencias}

Acosta, K. (2007). Elaboración de una pasta alimentaria a partir de sémola de diferentes variedades de cebada. (Tesis de licenciatura). Universidad Autónoma del Estado de Hidalgo, Pachuca de Soto.

Araya, H., Park, N., Vera, G. and Alviña, M. (2003). Digestion rate of legume carbohydrates and glycemic index of legume-based meals. International Journal Food Sciences and Nutrition, 54(2), 119-126.

Asaduzzaman, K. and Mousumi, T. (2012). Nutritional and Medicinal Importance of Pleurotus Mushroom: An Overview. Food Reviews International, 28(3), 313-329.

Association of Official Analytical Chemists [AOAC]. (1990). Official Methods of Analysis (15 $5^{\text {th }}$ ed.). Washington, United States: Association of Official Analytical Chemists.

Astaíza, M., Ruiz, L. y Elizalde, A. (2010). Elaboración de pastas alimenticias enriquecidas a partir de harina de quinoa y zanahoria. Revista Biotecnología en el Sector Agropecuario y Agroindustrial, 8(1), 43-53.

Cardinale, A., Bourges, H. y Rivera, P. (2005). Pasta es salud. México: Comunica-t.

Food and Agriculture Organization [FAO]. (2020). The state of agricultural commodity markets 2020. Agricultural markets and sustainable development: Global value chains, smallholder farmers and digital innovations. Rome, Italy: Food and Agriculture Organization.

Food and Agriculture Organization [FAO], International Fund for Agricultural Development [IFAD], United Nations Children's Emergency Fund [Unicef], World Food Programme [WFP] and World Health Organization [WHO]. (2020). The state offood security and nutrition in the world 2020. Transforming food systems for affordable healthy diets. Rome, Italy: Food and Agriculture Organization. Retrieved from https://doi.org/10.4060/ca9692en.

Ghorai, S., Banik, S. P., Verma, D., Chowdhury, S., Mukherjee, S. and Khowala, S. (2009). Fungal biotechnology in food and feed processing. Food Research International, 42(5-6), 577-587.

Guzmán, G., Mata, G., Salmones, D., Soto, C. y Guzmán, L. (2008). El cultivo de los hongos comestibles. Ciudad de México, México: Instituto Politécnico Nacional.

Laboratorio Profeco Reporta. (2017). Soltamos la sopa. Revista del Consumidor, 480, 32-44. 
Lau, O. (1983). Methods of chemical analysis of mushrooms. In Chang, S. T. and Quimio, T. H. (eds.), Tropical mushrooms: biological nature and cultivation methods (pp. 87116). Hong Kong, China: The Chinese University Press.

Lavelli, V., Proserpio, C., Gallotti, F., Laureati, M. and Pagliarini, F. (2018). Circular reuse of bio-resources: The role of Pleurotus spp. in the development of functional foods. Food and Function, 9(3), 1353-1372.

La Moderna. (1 de octubre de 2019). La Moderna celebra el Día Mundial de la Pasta. Comunicado de prensa. Recuperado de https://www.lamoderna.com.mx/nosotros/prensa/2019/67-dia-mundial-pasta-lamoderna.

Mattila, P., Salo, P., Konko, K., Aro, H. and Jalava, T. (2002). Basic composition and amino acid contents of mushrooms cultivated in Finland. Journal of Agricultural and Food Chemistry, 50(22), 6419-6422.

Ng, S. H, Wan Amir Nizam, W. A. and Wan Rosli, W. I. (2017). Incorporation of Pleurotus sajor-caju powder in cinnamon biscuit: study on nutritional, physical, colour and sensorial properties. International Food Research Journal, 24(6), 2442-2450.

Parab, D. N., Dhalagade, J. R., Sahoo, A. K. and Ranveer, R. C. (2012). Effect of incorporation of mushroom (Pleurotus sajor-caju) powder on quality characteristics of Papad (Indian snack food). International Journal of Food Sciences and Nutrition, 63(7), 866-870.

Proserpio, C., Lavelli, V., Laureati, M. and Pagliarini, E. (2019). Effect of Pleurotus ostreatus powder addition in vegetable soup on $\beta$-glucan content, sensory perception, and acceptability. Food Science and Nutrition, 7(2), 730-737.

Rampinelli, J. R., Silveira, M. L., Gern, R. M., Furlan, S. A., Ninow J. L. and Wisbeck, E. (2010). Nutritional value of Pleurotus djamor cultivated in banana straw. Alimentos $e$ Nutrição Araraquara, 21(2), 197-202.

Secretaría de Economía. (23 de septiembre de 2002). Declaratoria de vigencia de la Norma Mexicana NMX-F-023-NORMEX-2002. Diario Oficial de la Federación. Recuperado http://diariooficial.gob.mx/nota_detalle.php?codigo=721062\&fecha=23/09/2002.

Serralda, A., Meléndez, G. y Pasquetti, A. (2003). Requerimientos y recomendaciones proteicas, referencias internacionales y mexicanas. Revista de Endocrinología y Nutrición, 11(2), 73-79. 
Soto, C., Álvarez, I. Martínez, C. y Ruiz, M. (2016). Elaboración de un pan de caja adicionado con harina del hongo Pleurotus spp. y cuantificación de aminoácidos y aspectos reológicos. Ponencia presentada en el Congreso Internacional de Investigación Academia Journals en Ciencias y Sustentabilidad 2016. Tuxpan, del 28 al 30 de septiembre de 2016.

Soto, C. y Arias, A. (2004). El cultivo de las setas (Pleurotus spp.). Una tecnología de producción de alimentos. Zapopan, México: Cuellar.

Soto, C., Serratos, J. C., Ruiz, M. y García, P. (2005). Análisis proximal y de aminoácidos de los residuos de cosecha del hongo Pleurotus spp. Revista Mexicana de Micología, (21), 49-53.

Vega, A. y Franco, H. (2012). Productividad y calidad de los cuerpos fructíferos de los hongos comestibles Pleurotus pulmonarius y $P$. djamor cultivados sobre substratos lignocelulósicos. Información Tecnológica, 24(1), 69-78. 


\begin{tabular}{|l|l|}
\hline ROL DE CONTRIBUCIÓN & AUTOR (ES) \\
\hline Conceptualización & Conrado Soto Velazco \\
\hline Metodología & Conrado Soto Velazco (igual) y Celia de la Mora Orozco (igual) \\
\hline Software & No aplica \\
\hline Validación & Conrado Soto Velazco (igual) e Isela Alvarez Barajas (igual) \\
\hline Análisis Formal & Celia de la Mora Orozco (principal) e Isela Alvarez (apoyo) \\
\hline Investigación & Celia de la Mora Orozco \\
\hline Recursos & Conrado Soto Velazco \\
\hline Curación de datos & Isela Alvarez Barajas \\
\hline $\begin{array}{l}\text { Escritura - Preparación del } \\
\text { borrador original }\end{array}$ & Celia de la Mora Orozco \\
\hline Escritura - Revisión y edición & Conrado Soto Velazco \\
\hline Visualización & Conrado Soto Velazco \\
\hline Supervisión & Conrado Soto Velazco \\
\hline Administración de Proyectos & Conrado Soto Velazco \\
\hline Adquisición de fondos & Conrado Soto Velazco (principal) e Isela Alvarez Barajas \\
\hline
\end{tabular}

